\title{
Exact Static Cylindrical Black Hole Solution to Conformal Weyl Gravity
}

\author{
Jackson Levi Said*, ${ }^{1}$ Joseph Sultana ${ }^{\dagger},{ }^{2}$ and Kristian Zarb Adami ${ }^{\ddagger 1,3}$ \\ ${ }^{1}$ Physics Department, University of Malta, Msida, MSD 2080, Malta \\ ${ }^{2}$ Mathematics Department, University of Malta, Msida, MSD 2080, Malta \\ ${ }^{3}$ Physics Department, University of Oxford, Oxford, OX1 3RH, United Kingdom
}

(Dated: 5 December 2018)

\begin{abstract}
We present the exact neutral black string solution in locally conformal invariant Weyl gravity. As a special case, the general relativity analogue still can be attained; however, only as a sub-family of solutions. Our solution contains a linear term that would thus result in a potential that grows linearly over large distances. This may have implications for exotic astrophysical structures as well as matter fields on the extremely small scale.
\end{abstract}

PACS numbers: 04.20.-q, 04.50.Gh

\section{INTRODUCTION}

There is a broad consensus in the community that Einstein's theory of general relativity describes well the behavior of gravitating bodies in the solar system. This arose partly because of the remarkable successes of general relativity in predicting the outcomes of the classical tests. However, when the galactic scale and beyond is considered, there has been a divide; in particular, the split can be classified into two broad categories. One method advanced is that of mass inference, where dark matter and then dark energy is placed in the galactic and then the cosmological scale such that the observations are an outcome of the theory using standard Newtonian dynamics. The other, which has received much attention in recent years, [1] is where the model of gravity is modified in some way such that either no or little dark matter is added. The proviso of these models is, however, that in the solar system scale and up to current testability, the theories must agree with general relativity in order to preserve the gains made thus far.

The choice of the Einstein-Hilbert action, which produces general relativity, is constrained by the requirement that the resulting equations of motion be no higher than second order. This renders field equations that are relatively simple compared with other theories, but which fail to describe observations on scales much higher than the solar system without placing a large amount of dark matter to account for the resulting galactic rotational curves. For this reason, a growing number of alternative actions are being pursued, some driven by observation, others by foundational development. One such model of the latter type is conformal Weyl gravity, introduced in Refs. 2-4]. This theory employs the principle of local conformal invariance of the spacetime manifold as the supplementary condition that fixes the gravitational action, instead of the requirement that the equations of motion be no higher than second order. This leads to fourth order equations of motion for the gravitational field. Nonetheless, the assumption of the local invariance principle, besides being in line with the way actions are chosen in field theory, leads to a unique action of conformal Weyl gravity among all other fourth order theory actions. Furthermore, this invariance principle may also provide a better link with the fundamental quantum nature of reality due to the added symmetry inherent in the model.

The outline of the paper is as follows. In Sec. II, we give an introduction of the field equations of conformal Weyl gravity with a brief review of the work done on the spherically symmetric case. In Sec. III, we derive a static and cylindrically symmetric solution to the field equations in Weyl gravity and compare it with the static cylindrically symmetric solution in general relativity obtained in Ref. [5]. Some characteristics of this new solution, such as the temperature and surface gravity of the cylindrical event horizon, are obtained in Sec. IV. Finally, we end in Sec. $\mathrm{V}$ with a discussion and some conclusions. Note that we use units where $G=1=c$.

\footnotetext{
*jsai0004@um.edu.mt

†joseph.sultana@um.edu.mt

${ }^{\ddagger}$ kristian.zarb-adami@um.edu.mt
} 


\section{CONFORMAL GRAVITY}

The action for general relativity is given by

$$
S=-\frac{1}{16 \pi} \int d^{4} x \sqrt{-g}(R-2 \Lambda),
$$

where the action for the observer is suppressed, $g$ is the determinant of the metric tensor, and $R$ is the Ricci scalar. Despite passing the classical tests and every observation to date, an issue naturally arises in the derivation of this action: Namely why should the condition that the resulting equations of motion be second-order guide the choice of action for gravity? That is, should not the order cutoff be chosen in accordance with some postulate of nature? One possible alternative, that when constrained appropriately can still comply with the classical tests, is one based on a local invariance principle. Besides the local gauge invariance to which general relativity is subject, we consider the singular restriction of local conformal invariance in choosing the action for our gravity theory. This means that the manifold $(\mathcal{M}, g)$ which emerges must be invariant to local stretchings [ $[$ ]

$$
g_{\mu \nu}(x) \rightarrow \tilde{g}_{\mu \nu}(x)=\Omega^{2}(x) g_{\mu \nu}(x)
$$

where $x$ represents spacetime positions on the manifold, and where the argument is not suppressed to emphasize that the conformal invariance takes place locally.

One of the immediate consequences of this postulate is that the artificially implanted cosmological constant, $\Lambda$, present in the action for general relativity in Eq.(11), must be withdrawn, since not to do so would introduce a length scale and thus break the conformal symmetry in the theory. However, as will be shown later, the same term naturally emerges out of the metric, which provides further circumstantial evidence for the effectiveness of the principle under consideration.

Turning now to the Weyl tensor

$$
\begin{aligned}
C_{\lambda \mu \nu \kappa} & =R_{\lambda \mu \nu \kappa} \\
& -\frac{1}{2}\left(g_{\lambda \nu} R_{\mu \kappa}-g_{\lambda \kappa} R_{\mu \nu}-g_{\mu \nu} R_{\lambda \kappa}+g_{\mu \kappa} R_{\lambda \nu}\right) \\
& +\frac{1}{6} R\left(g_{\lambda \nu} g_{\mu \kappa}-g_{\lambda \kappa} g_{\mu \nu}\right)
\end{aligned}
$$

which satisfies the conformal invariance condition 7]

$$
C_{\lambda \mu \nu \kappa} \rightarrow \tilde{C}_{\lambda \mu \nu \kappa}=\Omega^{2}(x) C_{\lambda \mu \nu \kappa}
$$

necessary to render a theory in the first place. Hence, this can be safely taken to be the unique Lagrangian density for Weyl gravity, since due to its very locally conformal invariant nature, it must be unique up to such transformations.

The consequence of this is that the Weyl action then becomes [3]

$$
\begin{aligned}
I_{W} & =\int d^{4} x \sqrt{-g} L \\
& =-\alpha \int d^{4} x \sqrt{-g} C_{\lambda \mu \nu \kappa} C^{\lambda \mu \nu \kappa} \\
& =-2 \alpha \int d^{4} x \sqrt{-g}\left[R_{\mu \nu} R^{\mu \nu}-\frac{1}{3} R^{2}\right]
\end{aligned}
$$

where the last equality is the simplest representation of the action, obtained by applying the Lanczos identity [8] to the Weyl tensor. Also, $\alpha$ is a dimensionless parameter which is usually chosen to be positive in order to satisfy the Newtonian lower limit. This parameter is the coupling constant of conformal gravity.

It has become quite popular recently to take the Einstein-Hilbert action present in Eq.(11) and add terms which then vanish as the scale of the phenomena is reduced so that they take place within the solar system. This method of generating extra fields that make part of gravity comes in various forms, among which are [9] $f(R)$ and [10] $f(T)$ gravity, where $T$ is the torsion scalar. However the distinction between these additional terms and Weyl gravity is that the action itself is different; that is, the driving force of change is the additional local conformal invariance postulate in the theory. Thus, instead of implanting a term in the action to explain new phenomenology at higher scales, in Weyl gravity the conformal constraint results as an outcome in the theory that only predicts divergences from Einstein-Hilbert theory when scales greater than the solar system are considered, closing at least one chapter in general relativity that of the order of the theory.

Taking now the variation of the action in Eq. (5) with respect to the metric leads to the field equations [6]

$$
\begin{aligned}
\sqrt{-g} g_{\mu \alpha} g_{\nu \beta} \frac{\delta I_{W}}{\delta g_{\alpha \beta}} & =-2 \alpha W_{\mu \nu} \\
& =-\frac{1}{2} T_{\mu \nu},
\end{aligned}
$$

where $T_{\mu \nu}$ is the stress-energy tensor and

$$
W_{\mu \nu}=2 C_{\mu \nu ; \alpha \beta}^{\alpha \beta}+C_{\mu \nu}^{\alpha \beta} R_{\alpha \beta},
$$


is the Bach tensor. Incidentally, due to the form of the field equations, whenever the Ricci tensor $R_{\mu \nu}$ vanishes, $W_{\mu \nu}$ also vanishes so that every vacuum solution of Einstein-Hilbert gravity also leads to a solution of Weyl gravity, and thus all the work done carries on into Weyl gravity naturally and without the need of alteration. Given, however, the increase in complexity in Weyl gravity, the converse does not automatically follow, meaning that not every vacuum solution from Weyl gravity implies a solution for general relativity, so that a new class of solutions are borne out.

This property emerges from the fact that $W_{\mu \nu}$ vanishes not only when the Ricci tensor vanishes, but also by other means, which can be seen by looking at Eqs.(6) and (7). Hence, the tensor that now characterizes the geometry, $W_{\mu \nu}$, will not exactly replace the Ricci tensor. Furthermore, in general, the fourth-order equations that make up $W_{\mu \nu}$ contain a several-fold increase in complexity for the field equations, which calls into question whether it will indeed be possible to find a solution with more generality than those found in general relativity.

For the task at hand and the problem addressed in this paper that of determining the vacuum cylindrical spacetime line element we consider the metric tensor in its most general form possible, $g_{\mu \nu}=\operatorname{diag}(-b(\rho), a(\rho), c(\rho), d(\rho))$, with the only further condition imposed that the spacetime is static, since we are interested currently in the simpler case of a neutral nonrotating spacetime.

At present, a number of conformal solutions have been found [3, 11 14, 20, 21, 23, 24] and studied in quite some detail. The case of spherical symmetry was studied by Mannheim and Kazanas in Ref.[3], where the initial problem seemed intractable but after a number of coordinate transformations a solution was indeed found. This exact static and spherically symmetric vacuum solution is given, up to a conformal factor, by the metric

$$
d s^{2}=-B(r) d t^{2}+\frac{d r^{2}}{B(r)}+r^{2}\left(d \theta^{2}+\sin ^{2} \theta d \phi^{2}\right),
$$

where

$$
B(r)=1-\frac{\beta(2-3 \beta \gamma)}{r}-3 \beta \gamma+\gamma r-k r^{2},
$$

and $\beta, \gamma$, and $k$ are integration constants. This solution encompasses the Schwarzschild solution $(\gamma=k=0)$ and the Schwarzschild-de Sitter solution $(\gamma=0)$ as special cases. In this solution, the parameter $\gamma$ measures the departure of Weyl theory from general relativity, and so for small enough $\gamma$, both theories have similar predictions. This parameter has dimensions of acceleration, and so Eq. (9) provides a characteristic, constant acceleration, which may be associated (in a non-obvious way) with the cosmological setting. Given the asymptotically non-flat character of the solution the parameter $\gamma$ has been associated [3] with the inverse Hubble length, i.e., $\gamma \simeq 1 / R_{H}$, which for a typical galaxy implies that the effects from the linear $\gamma r$ term in the metric become comparable to those due to the Newtonian potential term $2 \beta / r$ on distance scales roughly equal to the size of the galaxy a fact that led Mannheim and Kazanas to produce fits to the galactic rotation curves. The effect of the linear term $\gamma r$ in the metric on classical tests, particularly the bending of light, has been studied in detail in Refs. 15 19] The solution Eq.(9) was also generalized both to rotating and charged solutions in Ref.[11], heralding the complete generalization of spherical symmetry from general relativity into Weyl gravity.

Following the success of spherical symmetry in conformal gravity, research shifted to topological black holes, which culminated in Refs. 20, 21], where the question of conformal topological black holes was explored in general terms. Besides providing new solutions, these works showed that in conformal gravity, topological black hole solutions with non-negative scalar curvature $k$ at infinity are possible, unlike general relativity where only asymptotically anti-de Sitter (AdS) topological black holes are possible. The only exception is the toroidal case, $\mathcal{S}^{1} \times \mathcal{S}^{1}$, where the black hole interpretation is only possible for $k<0$ as in AdS gravity.

Besides compact spacetimes, cosmological effects have also been studied. Indeed, in Ref. 222] cosmological conformal gravity fluctuations were studied and the dark energy problem is discussed in this setting. In particular, the theory is put in a different setting so that it can be set against cosmological data, thus providing the way forward for further study into the local divergences from the isotropic and homogenous cosmos.

We now focus on the class of spacetimes in Weyl gravity with cylindrical symmetry. A number of analytic and numerical solutions were presented in Refs. 23, 24], including a generalization 
of the Melvin solution, as well as a study of the magnetic properties of conformal cylindrical solutions. However, the cylindrical solutions were obtained in a gauge that did not naturally generalize the well-known black string solution in general relativity, given by the Lemos metric [5]

$$
\begin{aligned}
d s^{2} & =-\left(\alpha^{2} r^{2}-\frac{b}{\alpha r}\right) d t^{2}+\frac{d r^{2}}{\alpha^{2} r^{2}-\frac{b}{\alpha r}} \\
& +r^{2} d \phi^{2}+\alpha^{2} r^{2} d z^{2}
\end{aligned}
$$

with the coordinate ranges

$$
\begin{aligned}
& -\infty<t<\infty, 0 \leq r<\infty, 0 \leq \phi<2 \pi \\
& -\infty<z<\infty
\end{aligned}
$$

and with $\alpha=\sqrt{-\frac{\Lambda}{3}}, b=M / 2$, and $M$ being mass.

Our aim is to find a Lemos-like black string solution in Weyl gravity similar to what Mannheim and Kazanas did in the spherically symmetric case discussed above, and then compare this with the Lemos metric to study further any similarities and differences between Weyl and Einstein's theories.

\section{THE CONFORMAL CYLINDRICAL METRIC}

In order to solve the conformal field equations with a cylindrically symmetric metric tensor, we consider a general line element in cylindrical coordinates $(t, \rho, \phi, z)$ :

$d s^{2}=-b(\rho) d t^{2}+a(\rho) d \rho^{2}+c(\rho) d \phi^{2}+d(\rho) d z^{2}$.

Since in cylindrical topology the background spacetime is not curved along the axial direction or over the angular coordinate, the metric elements will be independent of both $z$ and $\phi$. Moreover, since we are looking for a conformal generalization of the Lemos metric, Eq.(10), in the spirit of Ref.[3] , we can consider the Lemos gauge such that

$$
C(\rho)=\rho^{2} \quad \text { and } \quad D(\rho)=\alpha^{2} \rho^{2} .
$$

Following a similar procedure as in Ref. [3] , we reformulate the metric in order to reduce the computation required to solve the field equations.
The line element may be rewritten as

$$
\begin{aligned}
d s^{2} & =\frac{\rho^{2}(r)}{r^{2}}\left[-\frac{r^{2} b(r)}{\rho^{2}(r)} d t^{2}+\frac{r^{2} a(r) \rho^{\prime 2}(r)}{\rho^{2}(r)} d r^{2}\right. \\
& \left.+r^{2} d \phi^{2}+\alpha^{2} r^{2} d z^{2}\right],
\end{aligned}
$$

where $\rho(r)$ is an arbitrary function of $r$. Choosing this dependence such that

$$
\int \frac{d \rho}{\rho^{2}(r)}=-\frac{1}{\rho(r)}=\int \frac{d r}{r^{2} \sqrt{a(r) b(r)}},
$$

then yields

$$
\begin{aligned}
d s^{2} & =\frac{\rho^{2}(r)}{r^{2}}\left[-B(r) d t^{2}+A(r) d r^{2}+r^{2} d \phi^{2}\right. \\
& \left.+\alpha^{2} r^{2} d z^{2}\right]
\end{aligned}
$$

with $A(r)=1 / B(r)$ and $B(r)=\frac{r^{2} b(r)}{\rho^{2}(r)}$. The metric that results is thus conformally related to the standard general line element for cylindrical spacetimes with $A(r)=1 / B(r)$. Conformal transformations are allowed through Eq.(2), so we take

$$
g_{\mu \nu} \rightarrow r^{2} \rho^{-2}(r) g_{\mu \nu},
$$

and hence the general line element

$$
d s^{2}=-B(r) d t^{2}+\frac{d r^{2}}{B(r)}+r^{2} d \phi^{2}+\alpha^{2} r^{2} d z^{2},
$$

is formulated. Hence, the metric elements may now be determined up to an arbitrary overall $r$ dependent conformal factor. Furthermore, since vacuum solutions of $W_{\mu \nu}(\rho)$ will be considered, $W_{\mu \nu}(r)$ must also vanish, from which it follows that the information is completely transferred to $W_{\mu \nu}(r)$ so that this will contain all observable information in the vacuum case.

The method used in general relativity of calculating the Ricci tensors and then equating them to a vanishing stress-energy tensor becomes far too complicated in conformal gravity given Eq.(7). Indeed, this case strengthens when the covariant derivative is considered. Instead, as was done in Ref. [3] , we calculate the EulerLagrange equations using the generic line element

$d s^{2}=-B(r) d t^{2}+A(r) d r^{2}+r^{2} d \phi^{2}+\alpha^{2} r^{2} d z^{2}$,

which adopts the Lemos gauge for cylindrically symmetric spacetimes, and then substitute $A(r)=1 / B(r)$. 
The Euler-Lagrange equations turn out to be second order [3]

$$
\begin{aligned}
& \sqrt{-g} W^{\mu \mu}=\frac{\delta I}{\delta g_{\mu \mu}}=\frac{\partial}{\partial g_{\mu \mu}}(\sqrt{-g} \tilde{L}) \\
& -\frac{\partial}{\partial x^{\mu}}\left(\sqrt{-g} \frac{\partial \tilde{L}}{\partial\left(g_{\mu \mu}\right)^{\prime}}\right)+\frac{\partial^{2}}{\partial\left(x^{\mu}\right)^{2}}\left(\sqrt{-g} \frac{\partial \tilde{L}}{\partial\left(g_{\mu \mu}\right)^{\prime \prime}}\right)
\end{aligned}
$$

where 'indicates differentiation with respect to $r$ and $\tilde{L}=R_{\mu \nu} R^{\mu \nu}-R^{2} / 3$. We consider only the diagonal elements of $W^{\mu \nu}$ which only vary with respect to the radial coordinate since the line element in Eq.(19) has elements that depend only on that coordinate. The previous variation is taken for $\delta I / \delta A$ and $\delta I / \delta B$, respectively, yielding

$$
\begin{aligned}
\sqrt{\alpha^{2} r^{4} A B} W^{r r} & =-\frac{\alpha^{2}}{48 A(r)^{4} B(r)^{3} \sqrt{r^{4} \alpha^{2} A(r) B(r)}}\left[-7 r^{2} B(r)^{2} A^{\prime}(r)^{2}\left(r B^{\prime}(r)-2 B(r)\right)^{2}\right. \\
& +2 r^{2} A(r) B(r)\left(2 B(r)-r B^{\prime}(r)\right)\left(4 B(r)^{2} A^{\prime \prime}(r)+3 r A^{\prime}(r) B^{\prime}(r)^{2}\right. \\
& \left.-2 B(r)\left(r A^{\prime \prime}(r) B^{\prime}(r)+A^{\prime}(r)\left(2 r B^{\prime \prime}(r)+B^{\prime}(r)\right)\right)\right) \\
& +A(r)^{2}\left(-7 r^{4} B^{\prime}(r)^{4}+4 r^{3} B(r) B^{\prime}(r)^{2}\left(3 r B^{\prime \prime}(r)+5 B^{\prime}(r)\right)\right. \\
& +4 r^{2} B(r)^{2}\left(r^{2} B^{\prime \prime}(r)^{2}+B^{\prime}(r)^{2}-2 r B^{\prime}(r)\left(r B^{(3)}(r)+6 B^{\prime \prime}(r)\right)\right) \\
& \left.\left.+16 r B(r)^{3}\left(r\left(r B^{(3)}(r)+2 B^{\prime \prime}(r)\right)-2 B^{\prime}(r)\right)+16 B(r)^{4}\right)\right]
\end{aligned}
$$

and

$$
\begin{aligned}
\sqrt{\alpha^{2} r^{4} A B} W^{t t} & =\frac{-\alpha^{2}}{48 A(r)^{4} B(r)^{4} \sqrt{r^{4} \alpha^{2} A(r) B(r)}}\left[56 r^{3} B(r)^{3} A^{\prime}(r)^{3}\left(r B^{\prime}(r)-2 B(r)\right)\right. \\
& +r^{2} A(r) B(r)^{2} A^{\prime}(r)\left(57 r^{2} A^{\prime}(r) B^{\prime}(r)^{2}-4 r B(r)\left(13 r A^{\prime \prime}(r) B^{\prime}(r)\right.\right. \\
& \left.\left.+A^{\prime}(r)\left(19 r B^{\prime \prime}(r)+13 B^{\prime}(r)\right)\right)+4 B(r)^{2}\left(26 r A^{\prime \prime}(r)+7 A^{\prime}(r)\right)\right) \\
& +2 r A(r)^{2} B(r)\left(29 r^{3} A^{\prime}(r) B^{\prime}(r)^{3}-6 r^{2} B(r) B^{\prime}(r)\left(2 r A^{\prime \prime}(r) B^{\prime}(r)\right.\right. \\
& \left.+A^{\prime}(r)\left(9 r B^{\prime \prime}(r)+4 B^{\prime}(r)\right)\right)+4 r B(r)^{2}\left(A^{\prime}(r)\left(r\left(6 r B^{(3)}(r)+13 B^{\prime \prime}(r)\right)-5 B^{\prime}(r)\right)\right. \\
& \left.+r\left(4 r A^{\prime \prime}(r) B^{\prime \prime}(r)+\left(r A^{(3)}(r)+3 A^{\prime \prime}(r)\right) B^{\prime}(r)\right)\right) \\
& \left.+8 B(r)^{3}\left(2 A^{\prime}(r)-r\left(r A^{(3)}(r)+A^{\prime \prime}(r)\right)\right)\right)+A(r)^{3}\left(-16 r^{3}\left(4 B^{(3)}(r)+r B^{(4)}(r)\right) B(r)^{3}\right. \\
& +49 r^{4} B^{\prime}(r)^{4}-4 r^{3} B(r) B^{\prime}(r)^{2}\left(29 r B^{\prime \prime}(r)+11 B^{\prime}(r)\right) \\
& \left.\left.+4 r^{2} B(r)^{2}\left(9 r^{2} B^{\prime \prime}(r)^{2}-5 B^{\prime}(r)^{2}+2 r B^{\prime}(r)\left(6 r B^{(3)}(r)+13 B^{\prime \prime}(r)\right)\right)+16 B(r)^{4}\right)\right] .
\end{aligned}
$$

The other two elements, $W^{\phi \phi}$ and $W^{z z}$, do not

need to be taken into account, since we have a 
sufficient number of constraints. These two further equations provide us with an independent check of any solution that results.

We now restrict ourselves to the line element in Eq.(19), taking $A(r)$ to be the reciprocal of $B(r)$. This implies that we need only one constraint to determine the line element, since $B(r)$ turns out to be the only unknown. Hence, all the information is contained in $W^{r r}$, irrespective of the number of derivatives it contains. Taking the vacuum solution, $W^{r r}=0$, the resulting equation to solve becomes

$$
\begin{aligned}
& r^{2}\left(-r^{2} B^{\prime \prime}(r)^{2}-4 B^{\prime}(r)^{2}\right. \\
& \left.+2 r B^{\prime}(r)\left(r B^{(3)}(r)+2 B^{\prime \prime}(r)\right)\right) \\
& -4 r B(r)\left(r\left(r B^{(3)}(r)+B^{\prime \prime}(r)\right)-2 B^{\prime}(r)\right) \\
& -4 B(r)^{2}=0
\end{aligned}
$$

which can in principle be solved analytically.

In order to reduce the overall order of the differential equation, we transform $B(r)$ by

$$
B(r)=r^{2} l(r),
$$

which enables us to rewrite Eq. (23) as

$$
-r^{2} l^{\prime \prime}(r)^{2}+8 l^{\prime}(r)^{2}+2 r l^{\prime}(r)\left(r l^{(3)}(r)+4 l^{\prime \prime}(r)\right)=0 .
$$

hence reducing the order when the substitution

$$
l^{\prime}(r)=y(r)
$$

is taken. However since, ignoring derivatives, the function $l(r)$ appears twice in every term, we transform the plane by the exponential function

$$
y(r)=e^{f(r)},
$$

which surprisingly yields the relatively simple expression

$$
2 r^{2} f^{\prime \prime}(r)+r^{2} f^{\prime}(r)^{2}+8 r f^{\prime}(r)+8=0 .
$$

Again reducing the overall order of the differential equation through

$$
f^{\prime}(r)=h(r)
$$

so that the arbitrary function is constrained by

$$
2 r^{2} h^{\prime}(r)+r^{2} h(r)^{2}+8 r h(r)+8=0,
$$

the first integral is then

$$
h(r)=\frac{1}{a+\frac{r}{2}}-\frac{4}{r}
$$

and repeating each substitution in reverse we arrive at the final unknown in the line element in Eq.(19):

$$
B(r)=\frac{4 a^{2} c}{2 r}+2 a c+c r+d r^{2},
$$

where $a, c$, and $d$ are constants of integration.

The result in Eq. (32) has all the terms of the Lemos analogue; however, due to the placement of the constants, the exact form of the Lemos line element in Eq.(10) cannot be recovered. For this, we take the transformation

$$
\begin{aligned}
& a=\sqrt{\frac{b_{1}^{2} b_{2} b_{3}-3 b_{4}}{b_{2} b_{3}},} \\
& c=\frac{b_{3}}{4} \\
& d=b_{2}^{2}
\end{aligned}
$$

thus solving the problem of a conformal generalization of the Lemos metric where $B(r)$ is

$$
\begin{aligned}
B(r) & =\frac{b_{1}^{2} b_{2} b_{3}-3 b_{4}}{3 b_{2} r}+\sqrt{\frac{b_{3}\left(b_{1}^{2} b_{2} b_{3}-3 b_{4}\right)}{4 b_{2}}} \\
& +\frac{b_{3} r}{4}+b_{2}^{2} r^{2}
\end{aligned}
$$

or more conveniently, in the form

$$
A^{-1}(r)=B(r)=\frac{\beta}{r}+\sqrt{\frac{3 \beta \gamma}{4}}+\frac{\gamma r}{4}+k^{2} r^{2}
$$

where

$$
\begin{aligned}
& \beta=\frac{b_{1}^{2} b_{2} b_{3}-3 b_{4}}{3 b_{2}} \\
& \gamma=b_{3} \\
& k=b_{2},
\end{aligned}
$$

from which we can regain the Lemos line element by setting the emergent conformal factor $\gamma$ to zero. Hence, the conformal Lemos-like metric can be found without taking any approximations at all. Moreover, the solution in Eq. (37) or Eq. (32) also satisfies the remaining vacuum field equations $W^{i i}=0$ for $i=t, \phi$, and $z$.

Given the metric components in Eq.(37), we compare the conformal line element with the same line element derived in general relativity in 
Eq.(10). This is achieved by setting the new constant conformal factor $\gamma$ to zero and relating the remaining components, which would imply that

$$
\begin{aligned}
& k=\alpha, \\
& \beta=-\frac{b}{\alpha},
\end{aligned}
$$

hence we recover the expected metric for general relativity [5].

Lastly, we close by considering the curvature of the cylindrical metric; in particular, the Ricci curvature invariant turns out to be given by

$$
\mathcal{R}=g^{\mu \nu} R_{\mu \nu}=-\frac{24 k^{2} r^{2}+3 r \gamma+2 \sqrt{3 \beta \gamma}}{2 r^{2}} .
$$

The simplicity of this expression stems from the fact that the only nonvanishing Ricci tensor components are on the diagonal and depend only on one of the spacelike coordinates simplifying many of the derivatives and sums. In a similar, way the Kretschmann scalar invariant that results also turns out to be remarkably simple:

$\mathcal{K}=R^{\lambda \mu \nu \sigma} R_{\lambda \mu \nu \sigma}=\frac{1}{2 r^{6}}\left[24 \beta^{2}+6 \beta \gamma r^{2}+\gamma^{2} r^{4}+\right.$
$\left.12 \gamma k^{2} r^{5}+48 k^{4} r^{6}+8 \sqrt{3 \beta \gamma} r\left(\beta+\frac{\gamma r^{2}}{4}+k^{2} r^{3}\right)\right]$.

Through the above scalar invariant, the physical singularity is found to be located at $r=0$.

\section{TEMPERATURE}

The Hawking temperature of the metric in Eq.(19) with $A(r)$ and $B(r)$ as defined in Eq.(37) may provide interesting insight into the quantum nature of the surrounding spacetime. The surface gravity that forms the underpinning of temperature by the relationship $T_{H}=\frac{\kappa}{2 \pi}$ is defined by the formula 25]

$$
\kappa^{2}=-\frac{1}{2}\left(\nabla^{\mu} \chi^{\nu}\right)\left(\nabla_{[\mu} \chi_{\nu]}\right),
$$

where $\chi^{\nu}$ is the Killing field generating the horizon $r_{h}$ where $B\left(r_{h}\right)=0$. This is given by

$$
r_{h}=\frac{1}{12 k^{2}}\left[-\gamma+\frac{\gamma^{2}-24 k^{2} \sqrt{3 b \gamma}}{\Sigma}+\Sigma\right],
$$

where

$$
\begin{aligned}
& \Sigma^{3}=-864 \beta k^{4}-\gamma^{3}+36 \gamma k^{2} \sqrt{3 \beta \gamma}+ \\
& 12 \sqrt{3 \beta k^{4}\left(1728 \beta k^{4}+\gamma^{3}-48 \gamma k^{2} \sqrt{3 \beta \gamma}\right)},
\end{aligned}
$$

In the case of metric Eq.(19), the surface gravity given by Eq. (45) gives

$$
\kappa=-\frac{\beta}{2 r^{2}}+\frac{\gamma}{8}+k^{2} r
$$

and so the temperature is found to be

$$
T_{H}=\frac{\kappa}{2 \pi}=\frac{1}{\pi}\left(-\frac{\beta}{4 r_{h}^{2}}+\frac{\gamma}{16}+\frac{k^{2} r_{h}}{2}\right) .
$$

For $\gamma=0$ this reduces to the general relativity result [5] as expected.

\section{CONCLUSION}

The first point to note about our solution is that it is a vacuum solution $\left(W_{\mu \nu}=0\right)$ with no cosmological constant term in the field equations, whereas the Lemos metric, which is retrieved by taking $\gamma=0$ in Eq.(37), is a solution of Einstein's field equations in AdS gravity. The constant $\gamma$ thus measures the departure of Weyl gravity from general relativity. Comparing Eq.(37) with the Lemos metric in Eq. (10), which is also a vacuum solution of conformal gravity, we note that $\beta$ must be related to the negative of the mass and $k=\alpha=\sqrt{-\Lambda / 3}$ is related to the cosmological constant.

General relativity is by design a strong field theory in that in the weak field, it asymptotes to Newtonian gravity. Conformal (Weyl) gravity, on the other hand, aims to derive a theory of gravity using no restrictions on the order of the field equations. Applying just the invariance principle in Eq. (2), one can still achieve the standard Newtonian phenomenology in the weak field limit, [14] and possibly a solution to the dark matter and dark energy problems. The solution found in this paper will not be applicable to most astrophysical sources, since for the most part they are organized with spherical symmetry. On the other, hand for theories such as string theory, the new solution presented in this paper may have uses on scales just above the Planck scale [27]. However, in any case, detection of any such objects would in all likelihood be in the form of Hawking radiation, which is one possible 
avenue of future development for this metric. There may also be string-like applications in a number of other theories, such as with the use of the AdS/CFT correspondence duality.

In this paper, we derive the static cylindrically neutral metric for Weyl conformal gravity. The next step would be to generalize this to the whole family of charged rotating cylindrically symmetric solutions, as was done by Mannheim and Kazanas [11] for the Kerr-Neuman general relativity analogue. This adds to the current cylindrically symmetric solutions in Refs. [23, 24] in that the field equations are solved analytically in a gauge which naturally generalizes the black string solutions of Einstein's gravity.

\section{ACKNOWLEDGMENTS}

J. L. S. wishes to thank the Physics Department at the University of Malta for hospitality during the completion of this work.

[1] Clifton, T., Ferreira, P. G., Padilla, A., and Skordis, C., arXiv:1106.2476 2 [astro-ph.CO].

[2] Riegert, R. J., Phys. Rev. Lett. 53, 315 (1984).

[3] Mannheim, P. D., and Kazanas, D., Astrophys. J. 342, 635 (1989).

[4] Mannheim, P. D., and Kazanas, D., Astrophys. J. Suppl. 76, 431 (1991).

[5] Lemos, J. P. S., Phys. Lett. B 353, 46 (1995).
[6] DeWitt, B. S., Relativity, Groups and Topology, (New York: Gordon and Breach), 1964.

[7] Weyl, H., Math Zeit 2, 384 (1918).

[8] Lanczos, C., Ann. Math. 39842 (1938).

[9] Sotiriou, T. P., Faraoni, V., Rev. Mod. Phys. 82, 451 (2010).

[10] Garecki, J., arXiv:1010.2654v3.

[11] Mannheim, P. D., and Kazanas, D., Phys. Rev. D 44, 417 (1991).

[12] Mannheim, P. D., Gen. Relativ. Gravit. 22, 289 (1990).

[13] Mannheim, P. D., Astrophys. J. 391, 429 (1992).

[14] Mannheim, P. D., and Kazanas, D., Gen. Relativ. Gravit. 26, 337 (1994).

[15] Edery, A., and Paranjape, M. B., Phys. Rev. D 58, 024011 (1998).

[16] Pireaux, S., Class. Quantum Grav. 21, 1897, (2004).

[17] Pireaux, S., Class. Quantum Grav. 21, 4317, (2004).

[18] Amore, P., Arceo, S., and Fernandez, F. M., Phys. Rev. D 74, 083004, (2006).

[19] Sultana, J., and Kazanas, D., Phys. Rev. D 81, 127502, (2010).

[20] Klemm, D., Class. Quantum Grav. 15, 3195 (1998).

[21] Vanzo, L., Phys. Rev. D 56, 6475 (1997).

[22] Mannheim, P. D., arXiv:1109.4119v1 [gr-qc].

[23] Brihaye, Y., and Verbin, Y., Phys. Rev. D 81, 124022 (2010).

[24] Brihaye, Y., and Verbin, Y., Gen. Rel. Grav. 43, 2847 (2011).

[25] Jacobson, T., and Kang, G., Class. Quant. Grav. 10, L201 (1993).

[26] Mannheim, P. D. and O'Brien, J. G., arXiv:1011.3495 v3 [astro-ph.CO].

[27] Lemos, J. P. S., arXiv:gr-qc/0011092 v2. 